\section{Local application of biphosphonate as a biochemical barrier against bone graft resorption in mandibular reconstruction}

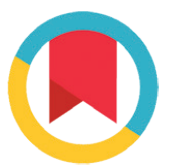

CrossMark

\author{
Romzi Hanif, Cahya Y. Hasan, ${ }^{*}$ Rahardjo, Muhammad M. Rahmat
}

\title{
Abstract
}

Objective: This paper was aimed at examining the positive results of iliac bone graft osteogenesis and osteointegration following the local application of bisphosphonate in mandibular reconstruction.

Methods: A 22-years-old female patient came with a complaint of pain-free right cheek mass that has enlarged slowly since 2 years. Radiographic examination shows cloudy radiolucent images in the right mandibular corpus to the right mandibular ramus, while histopathological examination indicates ossifying fibroma. Patient underwent mandibular resection followed by iliac bone graftbased mandibular reconstruction. Bone graft was immersed with bisphosphonate (zoledronate acid $0.005 \mathrm{mg} / \mathrm{ml}$ ) for 3 minutes, then rinsed with saline in 3 minutes, followed by fixing bone graft on the reconstruction plate.

Results: Postoperative follow up in the 36th week showed no signs of infection and dehiscence in the surgery site, and the radiographic examination indicated signs of osteogenesis and osteointegration of mandibular bone graft.

Conclusion: Local application of biphosponate on bone graft promotes the favorable results of osteogenesis and osteointegration in mandibular reconstruction.
Department of Oral and Maxillofacial Surgery, Faculty of Dentistry, Gadjah Mada University, Bandung, Indonesia

*Correspondence to: Cahya Y. Hasan, Department of Oral and Maxillofacial Surgery, Faculty of Dentistry, Gadjah Mada University, Bandung, Indonesia yustisiahasan@ugm.ac.id

Received: 11 Maret 2019

Revised: 26 September 2019

Accepted: 20 Desember 2019

Available Online: 1 April 2021

Keywords: Biphosponate, Bone graft, Mandibular reconstruction, Osteogenesis, Osteointegration

Cite this Article: Hanif R, Hasan CY, Rahardjo, Rahmat MM. 2021. Local application of biphosphonate as a biochemical barrier against bone graft resorption in mandibular reconstruction. Journal of Dentomaxillofacial Science 6(1): 61-65. D0I: 10.15562/jdmfs.v6i1.929

\section{Introduction}

Mandible is an essential anatomical structure for physiological and aesthetic functions of humans. Therefore, any mandibular defects caused by tumors, malignancy, trauma, osteomyelitis, and osteoradionecrosis will considerably affect patient's quality of life. Hemimandibulectomy, a type of mandibular resection may result in mandible defects leading to mandibular dysfunctions. ${ }^{1}$ he mandibular defects after hemimandibulectomy require reconstructions to restore mandibular aesthetics and functions. ${ }^{2}$

Mandibular reconstruction can be done by using reconstruction plate and iliac bone-derived non-vascularized autogenous bone graft. ${ }^{3}$ The plate mostly used for mandibular reconstruction is a rigid plate that is applied along the inferior mandibular margin. ${ }^{4}$ Among the advantages of non-vascularized autogenous bone graft involving iliac bone include the availability in large quantities $\left(50-90 \mathrm{~cm}^{3}\right)$, shorter surgical and recovery period, no need for microvascular surgery skills, simultaneous surgery by 2 teams, minimal donor site morbidity, minimal patient's side scar, various form harvest (block or particulate, cortical and corticocancellous), while the disadvantages are weak osteogenesis and excessive resorption in defects over $6 \mathrm{~cm}$ caused by the origin of endochondral and cortico-cancellous morphology. 5,6
Biphosponate is a preparation for minimizing osteoclast activity when in contact with bone surfaces, especially in subjects with active resorption where biphosponate works as a biochemical barrier for bone resorption. Biphosponate may also inhibit resorption in bone graft. Studies involving experimental animals with biphosponate-locally applied bone graft showed the ability to protect graft from resorption without affecting other skeletal bones. ${ }^{7}$

Local application of biphosponate to bone graft succeeds in inhibiting excessive bone graft resorption and fostering new bone formation (osteogenesis). This article reports on clinical and radiological evaluations of bone graft osteogenesis after the local application of biphosponate to hemimandibulectomy-post mandibular reconstruction.

\section{Case Report}

A 23-year-old female patient came to the oral surgery department of Dr. Sardjito General Hospital to check her enlarged right cheek and numb right lip. Clinical examination shows a facial asymmetry; there was enlargement of the mandibular ramus area which was hard in consistency, and there was an area with an increase in excitatory threshold on the cheek of the inferior mandibular margin and paresthesia in 

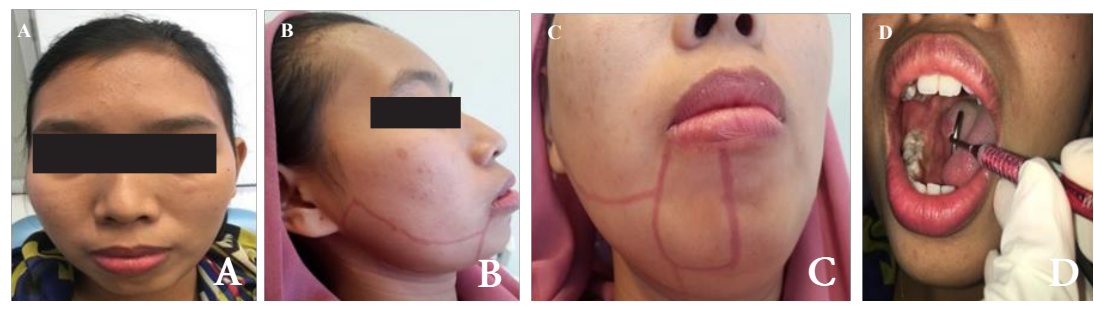

Figure 1 A. Facial asymmetry, B. Area with an increased excitatory threshold, C. Area of paresthesia, D. Enlargement of uneven-surfaced lingual bone of region 44 to 48
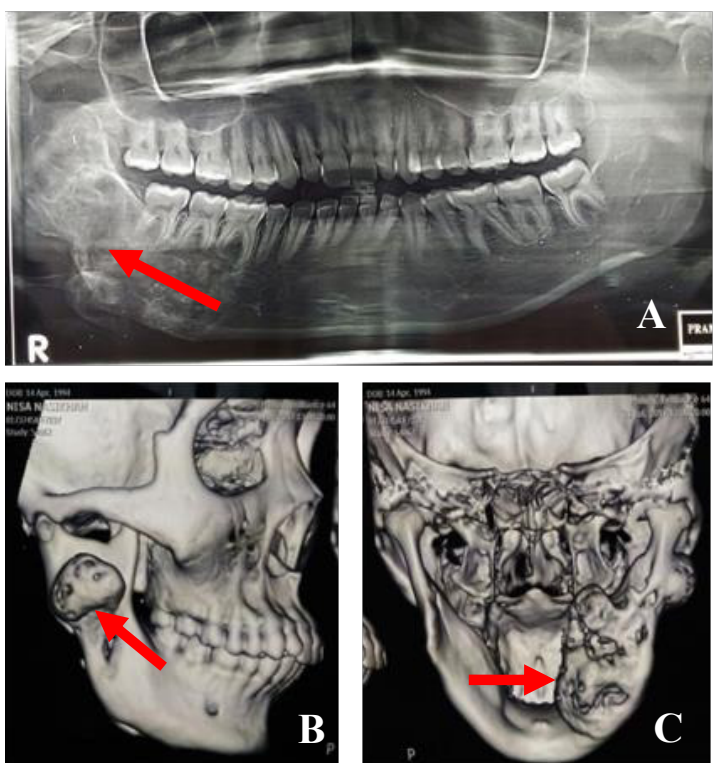

Figure 2 A. Diffuse cloud of smoke lesions on the ascending corpus-ramus in right mandibular angles (red arrow), B and C. Lesions of bone destruction in the lingual side of the corpus to the right ascending in the right mandible (red arrow)

the right lower lip The intraoral examination indicated an enlargement of the uneven- surfaced lingual bone of region 44 to 48 with the same gingival color as the surrounding tissue figure 1 .

The panoramic radiograph showed the presence of cloud of smoke in radiolucent lesions in the mandibular corpus region until the right mandibular ramus along with bone expansion and uneven angular inferior margin and resorption of tooth 46 and 47 roots 3D MSCT examination showed lesions in the mandibular ramus to the right mandibular subcondyle and bone destruction occurred in the corpus lingual side to the right mandibular ramus figure 2. The examination of anatomic pathology showed trabecularly- arranged bone tissue with osteoblastic rimming, corinective tissue stroma with proliferation of fibroblasts, which was declared as ossifying fibroma.
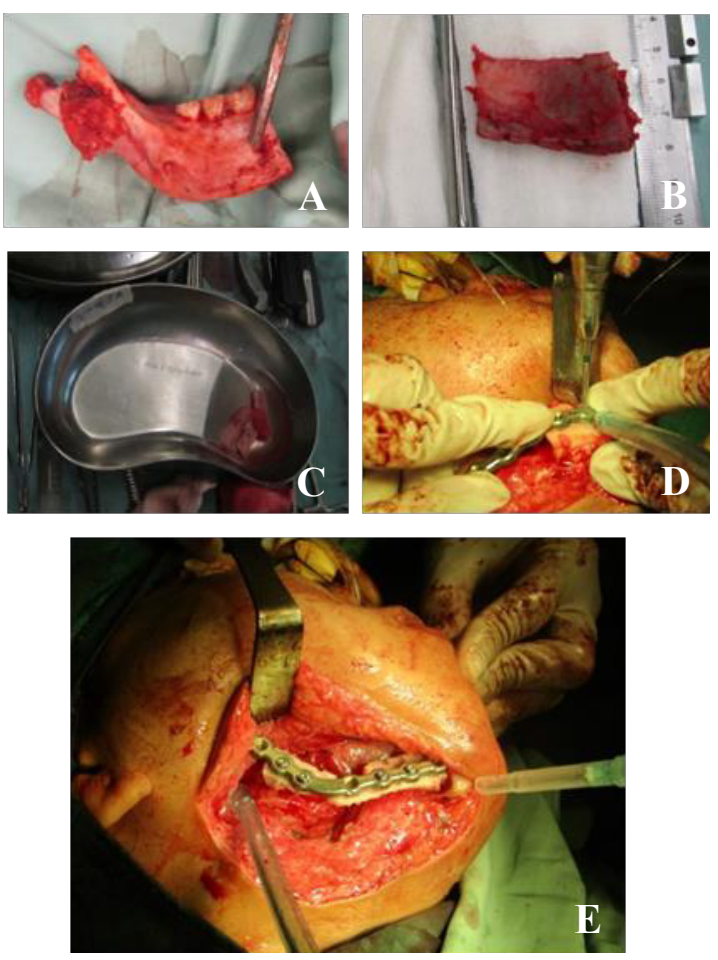

Figure 3 A. Hemimandibulectomy-post mandibular segment, B. Bicortical iliac bone graft, C. Iliac graft soaked in zometa for 3 minutes, subsequently rinsed with $0.9 \% \mathrm{NaCl}, \mathrm{D}$. Mounted bridging plate on healthy bone with a 3 -hole $2.4 \times 10 \mathrm{~mm}$ screw, E. Fitting iliac graft into the reconstruction plate

This case was diagnosed as right mandibular ossifying fibroma. Right mandibular hemimandibulectomy and mandibular reconstruction were chosen for the treatment involving reconstruction plate and non-vascularized autogenous bone graft derived from the iliac bone along with local application of biphosponate on the bone graft. The surgery was carried out in collaboration with the orthopedic department to harvest a $3 \mathrm{~cm} \times 5 \mathrm{~cm} \times 1.5 \mathrm{~cm}$ iliac graft.

Hemimandibulectomy was performed by cutting the mandible from the tooth region 44, followed by mandibular reconstruction with mounted reconstruction plate. Harvested Iliac graft was soaked in biphosponate preparation, zoledro-nate $0.005 \mathrm{mg} / \mathrm{ml}$ for 3 minutes, after which it was rinsed with saline. The graft was then fitted to the reconstruction plate with the cortical side facing the reconstruction plate figure 3. Patients underwent inpatient post-operative treatment for 4 days and intermaxillary wiring was fixed and retained for 6 weeks.

Clinical evaluation on week 6 following the removal of the intermaxillary wiring showed no dehiscence and signs of intraoral and extraoral 

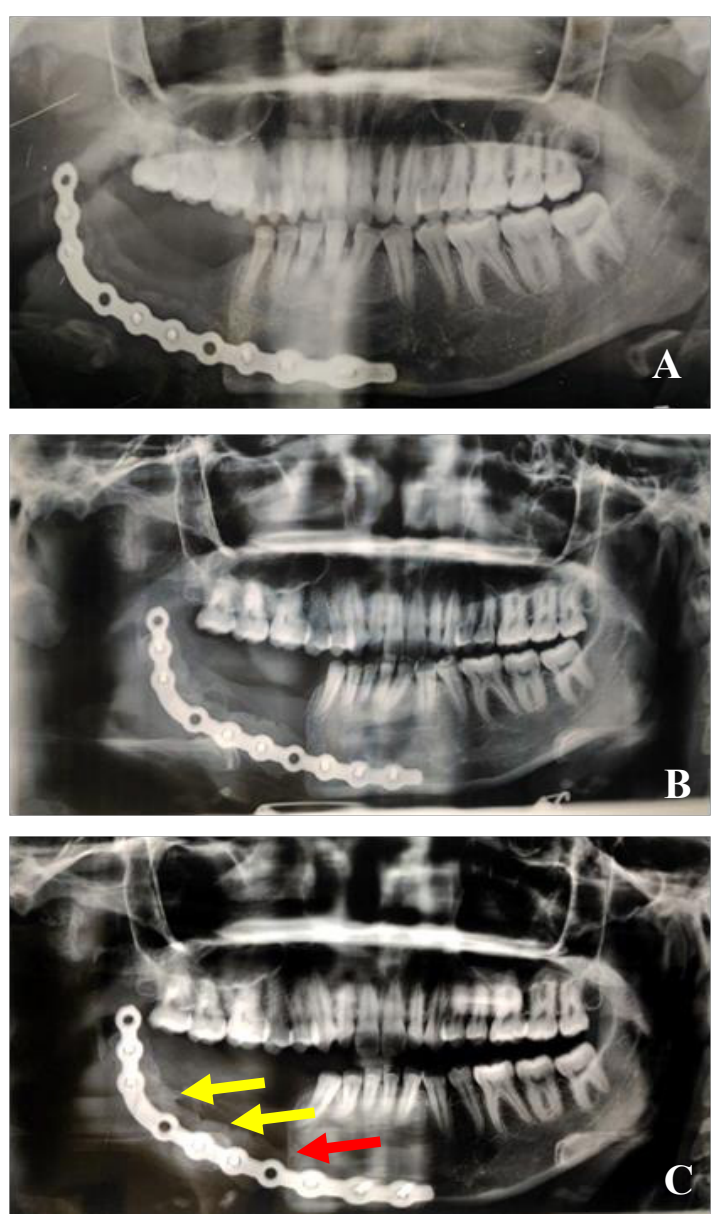

Figure 4 A. 12th week mandibular reconstructionpost panoramic $\mathrm{x}$-ray, B. 24th week mandibular reconstruction postpanoramic $x$ - ray, C. In 36th week mandibular reconstruction post panoramic $\mathrm{x}$-rayappearsosteointegration in the mandibular bone with graft (red arrow) and signs of osteogenesis along the graft (yellow arrow)

infection and left side occlusion was well formed. Evaluation of panoramic radiographs on postoperative week 12 and 24 showed a reconstruction plate was fitted to the right mandible and a graft image along the reconstruction plate with minimal graftresorption was seen. In postoperative week 36, evaluation of the panoramic radiograph was performed resulting in signs of osteointegration in the mandibular bone with graft in the distal area of tooth 43 , and signs of osteogenesis were apparent along the graft mounted to the reconstruction plate figure 4.

\section{Discussion}

Hemimandibulectomy is highly likely to result in mandibular defects. Mandibular reconstruction to restore the patient's anatomical function and shape must be done immediately after resection. ${ }^{8,9}$ Hemimandibulectomy, the radical resection along with reconstruction was opted for this case by considering lesions with the involvement of the lingual and buccal sides from the mandibular corpus to the right mandibular ascending ramus.

Ossifying fibroma is a benign tumor that belongs to a type of fibro-osseous lesion. Women, in their twenties, are more prone to this fibroma than men. Ossifying fibroma is most commonly found in the mandible especially in the posterior area or molar region $10 .^{10}$

Ossifying fibroma is slow but persistent in nature and causes expansion in the thinning of the buccal and lingual cortical layers to the lower mandibular inferior margin. This fibroma triggers no pain but paresthesia when occurring in the nerve structures. The oral epithelium will remain intact unless there is a secondary infection. ${ }^{10-12}$

Lesion of ossifying fibroma must be completely excised from the surrounding bone because of the high recurrence risk and proneness to malignancy. ${ }^{13}$ The possible method for treating ossifying fibroma is surgical excision for small-sized lesions with clear boundaries, whereas the large ones require radical surgery by involving the boundaries of surrounding healthy tissue and reconstruction. ${ }^{11,14}$ Hemimandibulectomy-based radical resection along with reconstruction were chosen to fit the condition of bone destruction and recurrence risk.

The opted method of mandibular reconstruction was a titanium plate, with non-vascularized autogenous bone graft derived from the iliac bone. Mandibular reconstruction plates and screw are the most widely used alloplastic materials for reconstructing the mandibular defects. The method is an easy, reliable procedure, and can be used for subsequent rehabilitation. ${ }^{15,16}$

Iliac bone serves as a graft donor for this case because it is numerous and relatively low in morbidity. Given that iliac bone is far from the facial region, it is made possible two teams operating simultaneously for efficient surgery. ${ }^{17}$

Prior to implantation, iliac graft bone was measured and shaped to fit into the reconstruction plate. The iliac graft was immersed in zometa solution $0.005 \mathrm{mg} / \mathrm{ml}$ for 3 minutes, after which it was rinsed with normal saline.

Zometa is a trademark for biphosponate preparation which contains zoledronate, a part of amino biphosponate, with nitrogen in the atomic chain of atoms and molecules. pembentukan tulang baru (osteogenesis) pada bone graft. The local application of zoledronate-derived biphosponate is expected to inhibit the excessive bone graft recession to occur before osteogenesis during bone graft. Local application of zelodronate $0.005 \mathrm{mg} / \mathrm{ml}$ 
in bone graft was done by immersing the graft for 3 minutes, followed by rinsing for 3 minutes in the experimental animal and resulted in slow resorption in the bone graft and an increase in new bone formation around the graft. ${ }^{18}$

Nitrogen-containing biphosponates such as pamidronate, olpadronate, ibandronate, alendronate, risedronate and zoledronate have a working mechanism that inhibits farnesyl diphosphate synthesis which causes failure of prenylation (fatty acid chain transfer) of various intracellular proteins, especially small GTP binding protein (Trimeric G-protein). Prenylation failure will trigger a premature apoptotic process of osteoclast cells resulting in inability for bone resorption. ${ }^{18,19}$

Experimental studies of bone graft locally applied with biphosponate showed the ability to protect graft from resorption without any effects on other skeletal bones. ${ }^{7}$ Locally applied biphosponate with zometa (zoledronate $0.005 \mathrm{mg} / \mathrm{ml}$ ) for 3 minutes in the experimental dog resulted in an increase in new bone formation in the group applied with zometa compared to the control group. ${ }^{7,18,20}$ Local application of biphosponate to alveolar bone defect was able to minimize alveolar bone resorption and improve new bone formation in the alveolar defect and foster the regeneration capacity of biomaterials and bone density.

Topical application of amino-biphosponate solution to bone defects or extraction-post socket, either alone or combined with a bone graft, is a risk-free procedure as biphosponate contributes to the initial phase of bone healing and is absorbed mainly by the attached bone so that tiny amount may be released into circulation. ${ }^{20,21}$

Studies on local application of biphosponate in humans are few. During the previous study, it was done by immersing the allograft bone in Bonefos (disodium clodronate) $10 \mathrm{ml}$ in a dose of $60 \mathrm{mg} /$ $\mathrm{ml}$ for 3 minutes then rinsed with normal saline $500 \mathrm{ml}$ in pelvic revision surgery. The postoperative 24th month control showed that rinsing of the morselized bone graft soaked in biphosponate could prevent resorption and minimize the risk of mechanical failure, whereas in the control group, the graft experienced resorption after 3 months. ${ }^{7,22}$

Radiographic evaluation of mandibular reconstruction-post bone graft in the 36th postoperative week showed minimal bone graft resorption and signs of osteogenesis along the surface of the bone graft and osteointegration occurred in the mandibular bone with graft in the distal area of tooth 43. The clinical evaluation indicated that locally-applied biphosponate was proven to minimize complications in the soft tissue or hard tissue around bone graft. ${ }^{23}$

\section{Conclusion}

Local application of biphosponate by immersing iliac bone graft in biphosponate preparations used in mandibular reconstruction-post hemimandibulectomy was proven to inhibit premature bone graft resorption, and increase osteogenesis along the bone graft surface and osteointegration of bone graft. The use of locally-applied biphosponate was proven to minimize complications in the soft tissue or hard tissue around bone graft.

\section{Acknowledgment}

Gratitude and appreciation are extended to all faculty members of the Department of Oral and Maxillofacial Surgery, Faculty of Dentistry, Universitas Gadjah Mada, Indonesia for good cooperation in the medication therapy management for this case study.

\section{Conflict of Interest}

The authors report no conflict of interest.

\section{References}

1. Hupp JR, Ellis E, Tucker MR. Contemporary oral and maxillofacial surgery 5th ed. St.Louis Missouri: Mosby Elsevier; 2008. p. 449-468.

2. Fernandes RP, Yetzer JG. Reconstruction of acquired oromandibular defect. Oral maxillofacial surg clin N Am 2013;25: 214-249.

3. Farwell DG, Futran ND. Oromandibular reconstruction. Facial Plast Surg 2000;16: 115-126.

4. Wong RC, Tideman H, Kin L, et al. Biomechanics of mandibular reconstruction: a review. IJOMS 2009;39: 313-319.

5. Chim H, Salgado CJ, Mardini S, et al. Reconstruction of mandibular defects. Plastic Surg 2010;24: 188-197.

6. Mounir M, Abou-ElFetouh A, El-Beialy W, et al. Vascularized versus non-vascularized autogenous bone graft for immediate reconstruction of segmental mandibular defects : a systematic review. OHDM 2015;14: 337-349.

7. Kesteris U, Aspenberg P. Rinsing morcellised bone grafts with biphosphonate solution prevents their resorption: a prospective randomized double-blinded study. J Bone \& Joint Surg 2006;88: 993-996.

8. Alfotawi R, Ayoub A. Reconstruction of maxillofacial bone defects: contemporary methods and future techniques. Am J Adv In Med Sc 2014;2: 18-27

9. Buchbinder D, Urken M. Mandibular reconstruction. Head \& Neck Surg- Otolaryngol 1993;2: 1980-1999.

10. Martín-Granizo R, Sánchez-Cuellar A, Falahat F. Cemento ossifying fibroma of the upper gingivae. Otolaryngol Head Neck Surg 2000;122: 775.

11. Triantafilidou K, Karakinaris D, Psomaderis $\mathrm{K}$, et al. Fibrous dysplasia of craniomaxillofacial bones a clinical study of 18 cases and review of the literature. BJDM 2014;18: 99-105.

12. Gondivkar SM, Gadbail AR, Chole R, et al. Ossifying fibroma of the jaws: report of two cases and literature review. Oral Oncol 2011;47: 804-809.

13. Toyosawa S, Yuki M, Kishino M, et al. Ossifying fibroma vs fibrous dysplasia of the jaw : molecular and immunological characterization. J Modern Pathol 2007;20: 389-396. 
14. Chang C, Hung H, Chang J. Central ossifying fibroma: clinicopathologic study of 28 case. J Formos Med Assoc 2008;7: 288-294.

15. Del-Hoyo JA, Sanroman JF, Bueno PR, et al. Primary mandibular reconstruction with bridging plates. J CranioMaxillofac Surg 1994;22: 43-48.

16. Stosic S. Mandibular reconstruction state of the art and perspectives. Vojnosanit Pregel 2008;65: 397-403.

17. Elsalanty ME, Genecov DG. Bone graft in craniofacial surgery. Craniomaxillofac Trauma Recons 2009;2: 125-134.

18. Hong C, Quach A, Lin L. Local vs sistemic administration of biphosphonate in rat cleft bone graft : a comparative study. BMJ 2018;10: 134-140.

19. Shaw NJ, Bishop NJ. Biphosphonate treatment of bone disease. Arch Dis Child 2005;90: 494-499.

20. Jakobsen T, Baas J, Bechtold J. The effect of soaking allograft in bisphosphonate. Clin Orthop Relat Res 2010;468: 867-874.
21. Lozano-Carrascal N, Salomo-Coll O, Hernandez-Alfaro F, et al. Do topical applications of biphosphonates improve bone formation in oral implantology? a systematic review. Med Oral Patol Oral Cir Bucal 2017;22: 512-519.

22. Mathijssen NM, Buma P, Hannink G. Combining biphosphonate with allograft bone for implant fixation. Cell Tissue Bank 2014;15: 3.

23. Widodo A, Rahmat MM, Prihartiningsih, et al. Inferior alveolar nerve preservation for hamimandibulectomy and bridging plate reconstruction in monostotic fibrous dysplasia case. J Dentomaxillofac Sci 2020;5: 196-199.

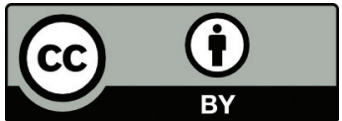

This work is licensed under a Creative Commons Attribution 